\title{
Rapid and Selective Crystallization of Acetaminophen using Metal-Assisted and Microwave-Accelerated Evaporative Crystallization
}

\author{
Muzaffer Mohammed ${ }^{1}$, Maleeha F Syed ${ }^{1}$, Mona J Bhatt ${ }^{1}$, Eugene J Hoffman ${ }^{2}$ and Kadir Aslan ${ }^{* 1}$ \\ ${ }^{1}$ Morgan State University, Department of Chemistry, 1700 East Cold Spring Lane, Baltimore, MD 21251 \\ ${ }^{2}$ Morgan State University, Department of Physics, 1700 East Cold Spring Lane, Baltimore, MD 21251 \\ * Corresponding author:kadir.aslan@morgan.edu
}

\begin{abstract}
In this paper, we demonstrate the application of Metal-Assisted and Microwave-Accelerated Evaporative Crystallization (MA-MAEC) technique to rapid and selective crystallization of a small drug compound. i.e. acetaminophen. Subsequent characterization of the crystals by optical microscopy, powder X-ray diffraction (PXRD) and Raman spectroscopy showed quantitatively selective growth of different crystal forms at various experimental conditions. Acetaminophen crystals were grown predominantly as Form I (99\%) on blank glass slides at room temperature. Form II crystals with 39\% purity grown on SIFs using microwave energy.
\end{abstract}

Keywords: Metal-Assisted and Microwave-Accelerated Evaporative Crystallization, Crystallization, Acetaminophen, Silver Nanoparticles.

Citation: M. Mohammed, et al. Rapid and Selective Crystallization of Acetaminophen using Metal-Assisted and Microwave-Accelerated Evaporative Crystallization. Nano Biomed. Eng. 2012, 4(1), 35-40.DOI: 10.5101/nbe.v4i1.p35-40.

\section{Introduction}

The determination of crystal structure of organic and biological molecules affords a better understanding of their behavior, both in-vivo and in-vitro [1, 2]. Various crystallization techniques are reported for the rapid growth of higher-quality crystals of organic and biological molecules [3-5] In this regard, Garetz et. al., employed polarized laser light irradiation to crystallize amino acids in solution [6]. Hamilton et. al. demonstrated the use of engineered surfaces such as nanoscale cylindrical pores in an effort to control polymorphism [7]. Myerson research group has developed crystallization platforms with selfassembled monolayers (SAMs) of alkane thiols for crystallization of biological and organic molecules using micro-droplet solvent evaporation [8]. More recently, Trout et. al. described a detailed investigation of the role of surface chemistry and morphology of porous polymer surfaces on heterogeneous nucleation of aspirin [9].

Previous work from our lab has demonstrated the use of Metal-Assisted and Microwave-Accelerated Evaporative Crystallization (MA-MAEC) technique as a quick and efficient method of growing crystals of amino acids with selective morphology $[10,11]$. MA-MAEC technique is based on the combined use of silver island films (SIFs) as a new crystallization platform and low power microwave heating to speed up the crystallization process. In MAMAEC, SIFs do not readily heat up when subjected to microwave heating, as compared to their surroundings and subsequently a temperature gradient between SIFs and their surroundings is created. The temperature gradient results in rapid mass transfer of molecules in solution towards the SIFs and amino acid molecules selectively assemble on to SIFs due to the strong affinity of silver nanoparticles towards amine groups [11].

Acetaminophen is a widely used over-thecounter analgesic and anti-pyretic drug [12]. Haisa et. al. identified two different crystalline forms of acetaminophen, Form I and Form II [13]. Form I crystals are monoclinic and are the most thermodynamically stable form of acetaminophen at room temperature, but they display poor compressibility. This was attributed to the absence of slip planes in the crystal structure which are necessary for a molecule to undergo plastic deformation 
during compression $[14,15]$. To overcome this deficiency, excipients are added to enhance compaction property and bind the molecules into the required shape. Form $\mathrm{I}$ is obtained by recrystallization of acetaminophen from water, ethanol or methanol. Addition of excipients escalates both the time and cost of manufacture. Form II of the acetaminophen crystals are orthorhombic metastable at room temperature; thus conversion of Form II to Form I can be carried out by exposing Form II crystals to increased temperature. Form II crystals have well defined slip planes which make them preferable for direct compression without need of excipients or binding agents. [16] The growth of Form II crystals by slow evaporation from ethanol solution[13] has been elusive to the scientific community. More recently Form II crystals are grown consistently and on a bulk scale by fusion/melting of Form I or amorphous acetaminophen and subsequently using it as seed for obtaining Form II crystals [15]. Form III acetaminophen has also been isolated and studied. It is very unstable at room temperature and has been found to grow only in a physically constrained setup [16]. Lang et. al. has studied the selective production of acetaminophen on polymer heteronucleus [17]. Chadwick et al. has recently has described the detailed a new method for selecting crystalline substrates to control polymorphism of acetaminophen by heterogeneous nucleation [18].

In this communication, we describe the recrystallization of commercially available acetaminophen (4-acetamidophenol; paracetamol) from deionized (DI) water on glass and SIFs at room temperature and using microwave heating at different microwave power levels. Subsequent characterization of the crystals by optical microscopy, powder X-ray diffraction (PXRD) and Raman spectroscopy shows quantitatively selective growth of different crystal forms under various experimental conditions. When grown on SIFs using microwave energy, Form II crystals with $39 \%$ purity were obtained. Other studies, in contrast, report that Form II crystals obtained from water solutions are transformed to Form I due to the presence of residual water in their crystal lattice [13].

\section{Materials and Methods}

The supersaturated acetaminophen solution was prepared by dissolving $3.02 \mathrm{~g}$ of acetaminophen in $10 \mathrm{ml}$ deionized (DI) water. The initial temperature of the solution was adjusted to $\left(80{ }^{\circ} \mathrm{C}\right)$. In the room temperature crystallization procedure, $20 \mu \mathrm{L}$ of the acetaminophen solution was pipette onto the blank glass slides or SIFs and time taken for complete evaporation of the solvent was noted. In the microwave-accelerated crystallization procedure, glass slides and SIFs were subjected to microwave heating at power level (PL) 10 in a conventional microwave oven (Emerson, maximum power 700W microwave oven, Model: MW8784B) until the solvent was completely evaporated. The above steps were repeated for microwave PL 5 and PL 1. SIFs from the same batch were used for each experimental condition.

\section{Results and Discussion}

The MA-MAEC technique is based on the combined use of SIFs and low power microwave heating that results in selective crystallization of molecules of interest through selective nucleation on SIFs and rapid evaporation of the solvent. Subsequently, it is important to comment on the evaporative nature of the MA-MAEC technique with respect to its relevant tools (SIFs and low power microwave heating). Scheme 1-top depicts the experimental geometry and thermal conductivities of the materials used in the MA-MAEC technique. In this regard, $20 \mathrm{ul}$ of supersaturated aqueous solution of acetaminophen at $80{ }^{\circ} \mathrm{C}$ is placed on a glass slide or SIFs (at room temperature) is allowed evaporate at room temperature or by microwave heating. In the room temperature evaporative crystallization experiments, the complete evaporation of water on a blank glass slide takes up to $71 \mathrm{sec}$, where the heat is preferentially transferred from warmer water $80^{\circ} \mathrm{C}$ to colder blank glass and air. Due to the larger thermal conductivity of silver, when SIFs are present on the glass slide, heat is transferred to them at a much higher rate than it would be to a blank glass surface or to air, water. The temperature gradient (difference between the temperature of solution and the surface where the crystals are formed) in room temperature evaporative crystallization is $\sim 58^{\circ} \mathrm{C}$.

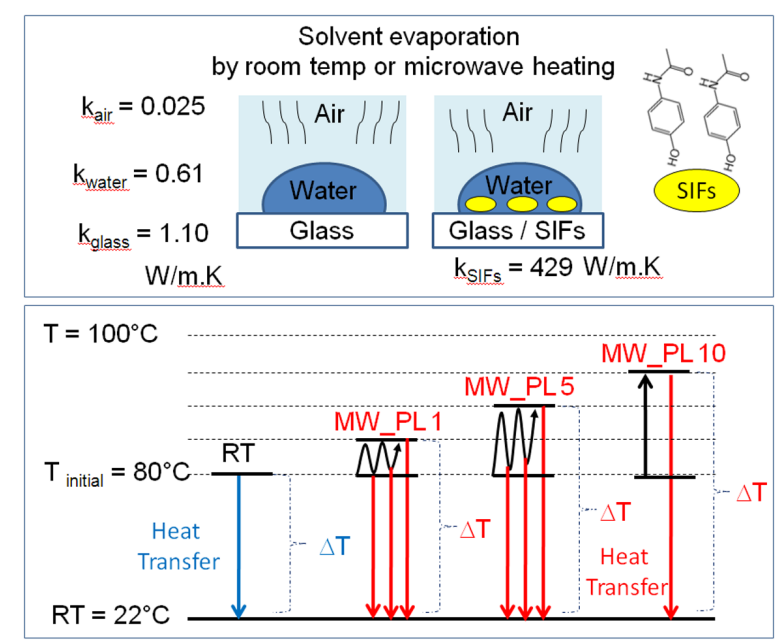

Scheme 1 Schematic depiction of (top) experimental geometry (bottom) temperature gradient created by initial heating of solution and additional heating by microwave heating. k-Thermal conductivity, W-Watts, K-Kelvin, T-Temperature, RT-Room Temperature, MW-Microwave heating, PL-Power level. $\Delta \mathrm{T}$-Temperature gradient

Although no direct evidence was obtained, the selective nucleation of acetaminophen crystals on SIFs is thought to be due the preferential interaction of functional groups of the acetaminophen (e.g. hydroxyl group- Scheme 1.) with SIFs, as predicted in a similar fashion to amino acids [10, 11] In this regard, acetaminophen is thought to assemble onto SIFs via hydroxyl groups and in combination with microwave heating affords for the selective nucleation of acetaminophen crystals. It is previously shown that molecules with hydroxyl functional groups assemble onto silver nanoparticles.[19] In contrast, the nucleation on 
glass slides (especially at room temperature) is thought to occur in a random fashion.

When a solution of acetaminophen identical to that described above is exposed to microwave heating, the temperature gradient between the solution and the surfaces increases depending on the employed duty cycle (Scheme 1-bottom). It is important to note that microwave power levels reported here correspond to the duty cycle of the microwave oven used in this work (power level 1 means full microwave power is on only $10 \%$ of the time, etc.). In all experiments carried out in this study, no visual evidence for the boiling of water was observed. Although not measured directly, it was concluded that the temperature of the acetaminophen solution never reached $100^{\circ} \mathrm{C}$. Since the microwave heating is on $100 \%$ of the time at microwave power level 10 , the solution is continuously heated $\left(<100^{\circ} \mathrm{C}\right)$ and the temperature gradient between the solution and the surfaces is maintained until water is completely evaporated. Subsequently, the larger temperature gradient results in faster evaporation of water and mass transfer of acetaminophen molecules towards the surface. At microwave power levels 1 and 5, microwave heating is on only $10 \%$ and $50 \%$ of the time, respectively, which results in intermittent cooling of the solution when the microwave heating is turned off. That is, the extent of the temperature gradient varies during the microwave heating at microwave power levels 1 and 5, and thus, the complete evaporation of water takes longer as compared to microwave heating at power level 10 .

\begin{tabular}{|c|c|c|c|}
\hline & & $\begin{array}{c}\text { Glass, DI water } \\
T_{\text {initial }}=80^{\circ} \mathrm{C}\end{array}$ & $\begin{array}{l}\text { SIFs, DI water } \\
T_{\text {initial }}=80^{\circ} \mathrm{C}\end{array}$ \\
\hline RT & $\begin{array}{c}\text { Time } \\
\% \text { Morphology } \\
\text { Size range }\end{array}$ & $\begin{array}{c}71 \pm 17 \mathrm{sec} \\
98 \% \text { Form } 1,2 \% \text { Form } 2 \\
140-453 \mu \mathrm{m}\end{array}$ & $\begin{array}{c}43 \pm 9 \mathrm{sec} \\
99 \% \text { Form } 1,1 \% \text { Form } 2 \\
755-1005 \mu \mathrm{m}\end{array}$ \\
\hline $\begin{array}{l}\mathrm{MW}_{-} \\
\mathrm{PL} 1\end{array}$ & $\begin{array}{c}\text { Time } \\
\% \text { Morphology } \\
\text { Size range }\end{array}$ & 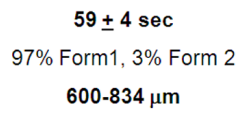 & 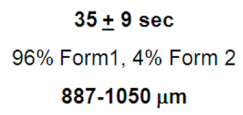 \\
\hline $\begin{array}{l}\mathrm{MW}_{-} \\
\mathrm{PL} 5\end{array}$ & $\begin{array}{c}\text { Time } \\
\% \text { Morphology } \\
\text { Size range }\end{array}$ & $\begin{array}{c}42 \pm 3 \mathrm{sec} \\
93 \% \text { Form } 1,7 \% \text { Form } 2 \\
739-1076 \mu \mathrm{m}\end{array}$ & $\begin{array}{c}10 \pm 0 \text { sec } \\
69 \% \text { Form } 1,31 \% \text { Form } 2 \\
671-2069 \mu \mathrm{m}\end{array}$ \\
\hline $\begin{array}{l}\text { MW_ } \\
\text { PL } 10\end{array}$ & $\begin{array}{c}\text { Time } \\
\% \text { Morphology } \\
\text { Size range }\end{array}$ & 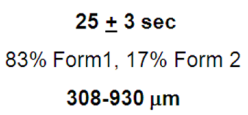 & $\begin{array}{c}\mathbf{1 5} \pm \mathbf{5} \mathbf{~ s e c} \\
61 \% \text { Form } 1,39 \% \text { Form } 2 \\
\mathbf{8 4 6 - 2 9 6 4 ~} \mu \mathrm{m}\end{array}$ \\
\hline
\end{tabular}

Table 1. Comparison of the time taken by acetaminophen in deionized water to completely crystallize on glass and Silver Island Films (SIFs) at room temperature and microwave heating power level (MW_PL) 1, 5, 10. DI-Deionized water. RT-Room Temperature. Each experiment was repeated 10 times. Sample size is 20 microliters.

Table 1 shows the comparison of the time taken by acetaminophen in DI water, to completely evaporate on glass and SIFs and the crystal morphology and size distribution of acetaminophen crystals formed at room temperature and by microwave heating at power level $1,5,10$. For a fixed volume of $20 \mu \mathrm{L}$ of acetaminophen solution, the total evaporation time on glass slides and SIFs at room temperature was recorded as 71 and $43 \mathrm{sec}$, respectively. That is, the use of silver nanoparticles in the crystallization process at room temperature reduced the total evaporation time by $\sim 2$-fold. When identical acetaminophen solutions were exposed to microwave heating, the total evaporation time was reduced up to 3- and 4-fold on both glass slides and SIFs. Moreover, evaporation using microwave heating was consistently faster on SIFs than glass slides. In this regard, the fastest complete evaporation occurred in $10 \mathrm{sec}$ on SIFs using microwave power level 5 (MW_PL 5).

Table 1 shows that the size of the acetaminophen crystals formed on glass at room temperature ranged between 140-453 $\mu \mathrm{m}$. The use of silver nanoparticles in the crystallization process at room temperature resulted in up to 7-fold increase in the size of acetaminophen crystals formed, where the size of acetaminophen crystals ranged between 755-1005 $\mu \mathrm{m}$. When identical acetaminophen solutions on glass and SIFs were exposed to microwave heating using microwave power level 1 , 5 and 10 , the crystal sizes were consistently increased for both substrates. With microwave power level 10 and SIFs, acetaminophen crystals 846 - $2964 \mu \mathrm{m}$ size were produced. This corresponds to a $\sim 22$-fold increase in size of acetaminophen crystals as compared to acetaminophen crystals produced using glass slides and room temperature evaporation.

Table 1 also shows that microwave heating of acetaminophen solutions significantly affects crystal morphology on both glass slides and SIFs. The acetaminophen crystals formed on glass at room temperature contained 98\% Form I and 2\% Form II (based on quantitative analysis of PXRD data, See Supporting Information). When identical acetaminophen solution on glass slides was exposed to microwave heating, the extent of Form I decreased from $97 \%$ for MW_PL 1 to $93 \%$ for MW_PL 5 and $83 \%$ for MW_PL 10 . On the other hand, the acetaminophen crystals formed on SIFs at room temperature contained 99\% Form I and $1 \%$ Form II. That is, the use of silver nanoparticles in the crystallization process at room temperature did not result in significant changes in crystal morphology. When identical acetaminophen solutions on SIFs were exposed to microwave heating, the extent of Form II increased from 4\% for MW PL 1 to $31 \%$ for MW PL 5 and 39\% for MW_PL 10. That is, one can produce Form II of acetaminophen crystals with $39 \%$ purity from aqueous solution by using SIFs and microwave heating.

Fig. 1 shows optical microscope images of acetaminophen crystals formed on blank glass slides and SIFs at room temperature and microwave heating. At room temperature, acetaminophen crystals formed on glass surface are predominantly Form I with a small number of crystals of Form II. Acetaminophen crystals formed on SIFs at room temperature appeared to be mostly Form II (We note that our PXRD results shows that Form II crystals were converted to Form I after 2 hours of crystal growth). When identical acetaminophen 
http://nanobe.org
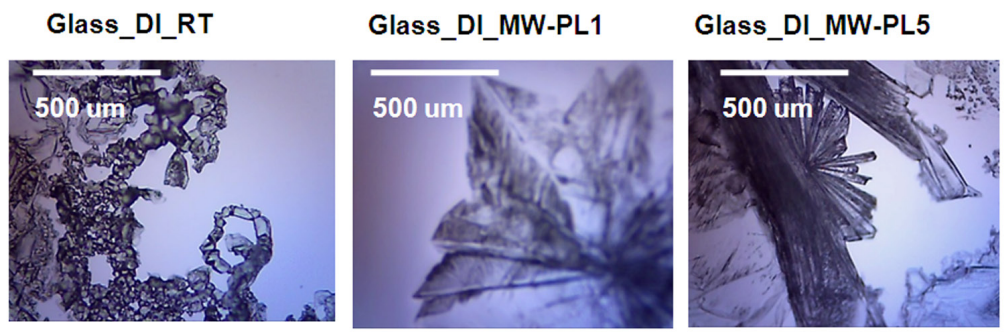

Glass_DI_MW-PL10

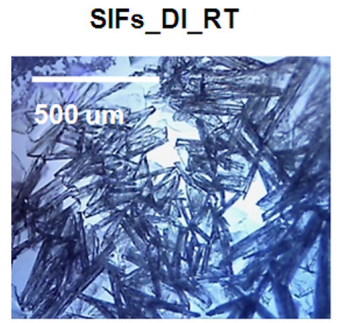

SIFs_DI_MW-PL1

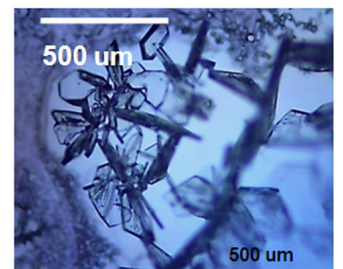

SIFs_DI_MW-PL5

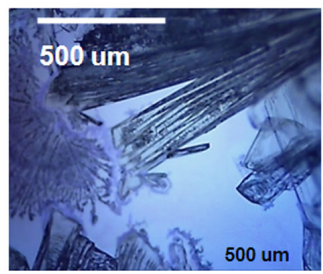

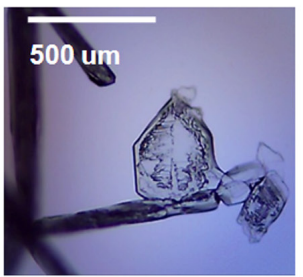

SIFs_DI_MW-PL10

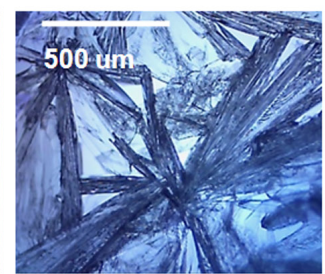

Fig. 1 Optical microscope images of acetaminophen crystallized from deionized (DI) water on blank glass slides and SIFs at room temperature and microwave heating. Form I crystals are monoclinic and appear as short prisms and plates, while Form II crystals are orthorhombic and have elongated needle-like shape.

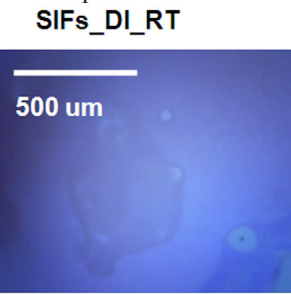

$0 \mathrm{sec}$

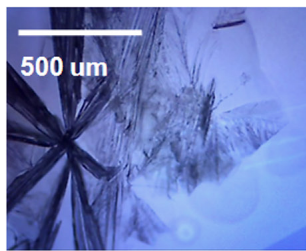

$20 \mathrm{sec}$

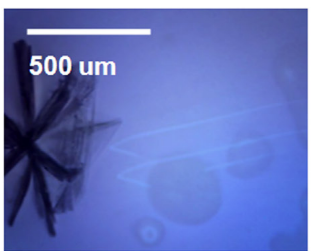

$5 \mathrm{sec}$

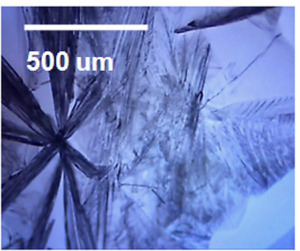

$25 \mathrm{sec}$

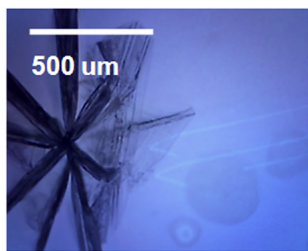

$10 \mathrm{sec}$

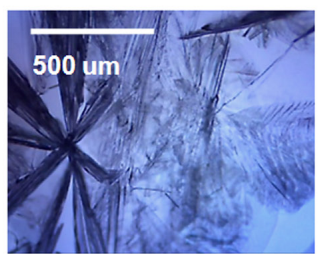

$30 \mathrm{sec}$

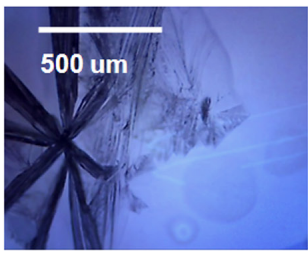

$15 \mathrm{sec}$

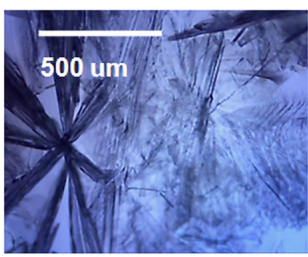

$35 \mathrm{sec}$

Fig. 2 Timed images of acetaminophen crystallized from deionized (DI) water on SIFs at room temperature. Please note that these times are for actual microwave heating and there is a $5 \mathrm{sec}$ delay in between each image used to collect the image.

solutions on blank glass slides are exposed to microwave energy at PL 1, 5, and 10, the extent of Form $\mathrm{I}$ is decreased while the extent of Form II is increased. Acetaminophen crystals formed on SIFs using microwave heating also contained Form II. Figure 1 also shows that the size of the acetaminophen crystals is significantly larger than those formed at room temperature on both blank glass slides and SIFs.

In order to visualize the growth of acetaminophen crystals during the evaporation of water at room temperature and using microwave heating, optical images of the solution and of the growing crystals on blank glass slides and SIFs were taken at time intervals as indicated in the Fig. 2 and Supplementary Figures S1-S6. In the experiments involving microwave heating glass slides and SIFs were taken removed from the microwave oven for a brief period of time $(\sim 5 \mathrm{sec})$ to collect optical images. Figure S1 shows the time progression of crystal growth on blank glass slides at room temperature. Crystals are not observed as the solution is deposited $(\mathrm{t}=0 \mathrm{sec})$, but within $10 \mathrm{sec}$ individual Form I crystals appear to assemble side-by-side and on top of one another to form a larger crystal structure. As water continues to evaporate, the large crystal assembly continues to grow ceasing after $70 \mathrm{sec}$ as the water is completely evaporated.

Fig. 2 shows the time progression of growth of acetaminophen crystals on SIFs at room temperature. Acetaminophen crystals appeared within 5 seconds after the placement of acetaminophen solution on SIFs. The crystal growth is clearly seen in the subsequent images, where the crystals seem to grow to their final size in 30-35 seconds. In these images, the darker acetaminophen crystals are Form II (and the surrounding opaque acetaminophen crystals are Form I. The size of acetaminophen crystals formed on SIFs at room temperature is significantly larger than those formed on blank glass slides at room temperature. This implies that one can grow larger and selective (Form II) acetaminophen crystals simply by incorporating silver nanostructures on to glass slides. 


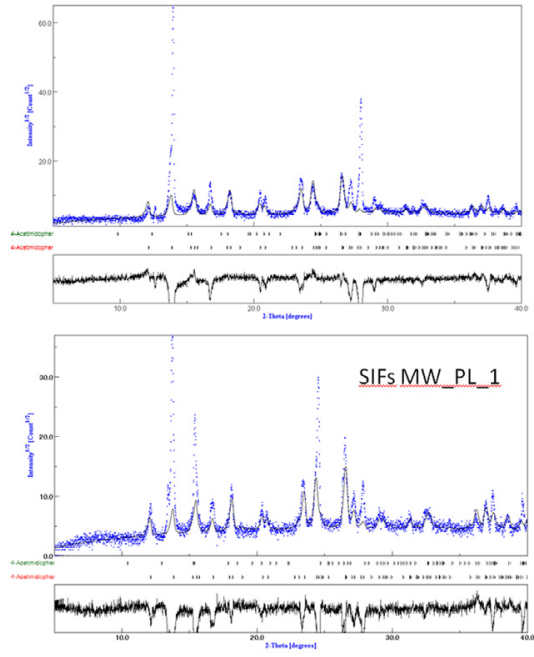

Fig. 3 Experimental PXRD data plotted using open-access software (Materials Analysis Using Diffraction or MAUD) for aceteminophen crystals form on SIF_RT and on SIFs_MW_PL10. The overlap shows the extent of matching of experimental data with standard data for acetaminophen (generated using ".CIF files" for acetaminophen obtained from Cambridge Crystallographic Data Center) and is used in quantitative analysis of the experimental data

Fig. 3 shows the plot of experimental XRD data for acetaminophen crystals grown on SIFs at room temperature and on SIFs with microwave heating (MW PL1, See Supp. Info Fig. S8-S9 for other conditions) with the overlapping standard XRD patterns for Form I and Form II acetaminophen crystals, which was for quantitative analysis of the experimental data. The results of the quantitative analysis of the experimental data were presented in Table 1. At first glance, one can notice that the experimental and the standard data for Form I and Form II match well.

It is important to note the crystal morphology was assessed by qualitatively by optical microscopy (immediately after or during crystallization) and quantitatively by PXRD (within 2 hours of crystallization). Our optical images show that Form II crystals are predominantly on SIFs at room temperature and with microwave heating. However, PXRD results show a significant reduction in Form II, which is attributed to the unstable nature of Form II acetaminophen crystals, as reported by others [13]. Nevertheless, our technique yielded up to $39 \%$ Form II even after 2 hours of the crystallization process.

\section{Conclusions}

In summary, we have presented the application of the MA-MAEC technique to rapid and selective crystallization of a small drug compound. i.e., acetaminophen. Acetaminophen crystals were formed predominantly as Form I (99\%) on blank glass slides at room temperature. On the other hand, the introduction of silver nanoparticles onto glass slides resulted in $\sim 2$-fold reduction in total evaporation time and growth of larger Form II acetaminophen crystals at room temperature. This was attributed to the multiple roles served by silver nanoparticles during crystallization process: 1) as selective nucleation sites 2) faster cooling of the initial acetaminophen solution due to a $\sim 400$-fold larger thermal conductivity of silver nanoparticles than of glass slides. Moreover, when acetaminophen solutions on glass slides and SIFs are microwave heated, there occurs up to a $\sim 3$-fold reduction in total evaporation time and growth of Form II acetaminophen crystals up to $39 \%$ purity within 2 hours of crystallization process. The characterization of acetaminophen crystals was confirmed by optical microscopy, Raman spectroscopy (Supporting Information, Figure S7) and powder XRD. Work is currently underway on the application of the MA-MAEC technique to other drug compounds; the results of these studies will be published in due course.

\section{Acknowledgements}

The project described was supported by Award Number 5-K25EB007565-05 from the National Institute of Biomedical Imaging and Bioengineering. The content is solely the responsibility of the authors and does not necessarily represent the official views of the National Institute of Biomedical Imaging and Bioengineering or the National Institutes of Health. The authors would like to thank Dr. Erdogan Ergican for the interpretation of XRD and Raman spectroscopy data.

\section{References}

1. Diao Y, et al. Surface Design for Controlled Crystallization: The Role of Surface Chemistry and Nanoscale Pores in Heterogeneous Nucleation. Langmuir. 2011; 27(9):5324-5334.http://dx.doi. org/10.1021/la104351k

2. Sarma B. et al. Solid forms of pharmaceuticals: Polymorphs, salts and cocrystals. Korean Journal of Chemical Engineering. 2011; 28(2): 315-322.http://dx.doi.org/10.1007/s11814-010-0520-0

3. Li XX. et al. The Factors during Protein Crystallization: A Review. Crystallography Reports. 2008; 53(7):1261-1266.http://dx.doi. org/10.1134/S1063774508070286

4. Fevotte G. In situ raman spectroscopy for in-line control of pharmaceutical crystallization and solids elaboration processes: A review. Chemical Engineering Research \& Design. 2007; 85(A7): 906-920.http://dx.doi.org/10.1205/cherd06229

5. Barrett P. et al. A review of the use of process analytical technology for the understanding and optimization of production batch crystallization processes. Organic Process Research \& Development 2005; 9(3): 348-355.http://dx.doi.org/10.1021/op049783p

6. Garetz BA, J Matic, and AS Myerson. Polarization switching of crystal structure in the nonphotochemical light-induced nucleation of supersaturated aqueous glycine solutions. Physical Review Letters. 2002 ; 89(17)http://dx.doi.org/10.1103/ PhysRevLett.89.175501

7. Hamilton BD. et al. Manipulating Crystal Orientation in Nanoscale Cylindrical Pores by Stereochemical Inhibition. Journal of the American Chemical Society. 2009; 131(7): 2588-2596.http://dx.doi. org/10.1021/ja807193s

8. Lee AY. et al. Crystallization on confined engineered surfaces: A method to control crystal size and generate different polymorphs. Journal of the American Chemical Society. 2005; 127(43): 1498214983.http://dx.doi.org/10.1021/ja055416x

9. Trout BL. et al. Surface Design for Controlled Crystallization: The Role of Surface Chemistry and Nanoscale Pores in Heterogeneous Nucleation. Langmuir. 2011; 27(9):5324-5334.http://dx.doi. org/10.1021/la104351k

10.Alabanza AM. and K Aslan. Metal-Assisted and MicrowaveAccelerated Evaporative Crystallization: Application to L-Alanine. Crystal Growth \& Design. 2011; 11(10): 4300-4304.http://dx.doi. org/10.1021/cg201018j

11.Pinard MA. and K Aslan, Metal-Assisted and MicrowaveAccelerated Evaporative Crystallization. Cryst Growth Des. 2010; 
10(11): 4706-4709.http://dx.doi.org/10.1021/cg101059c

12. Guggenheimer J, and PA Moore. The therapeutic applications of and risks associated with acetaminophen use A review and update. Journal of the American Dental Association. 2011; 142(1): 38-44.

13. Haisa, M., et al., The monoclinic form of $\mathrm{p}$ hydroxyacetanilide. Acta Cystallographica B. 1976; B32: 1283-1285.http://dx.doi. org/10.1107/S0567740876012223

14. Al-Zoubi, N., J.E. Koundourellis, and S. Malamataris, FT-IR and Raman spectroscopic methods for identification and quantitation of orthorhombic and monoclinic paracetamol in powder mixes. Journal of Pharmaceutical and Biomedical Analysis. 2002; 29(3): 459-467. http://dx.doi.org/10.1016/S0731-7085(02)00098-5

15. Szelagiewicz M. et al. In situ characterization of polymorphic forms the potential of Raman techniques. Journal of Thermal Analysis and Calorimetry. 1999; 57(1): 23-43.http://dx.doi.org/10.1023/ A:1010184805966

16. Kachrimanis K, DE Braun, and UJ Griesser. Quantitative analysis of paracetamol polymorphs in powder mixtures by FT-Raman spectroscopy and PLS regression. Journal of Pharmaceutical and Biomedical Analysis. 2007; 43(2): 407-412.http://dx.doi.org/10.1016/ j.jpba.2006.07.032
17. Lang M AL, Grzesiak, and AJ Matzger. The Use of Polymer Heteronuclei for Crystalline Polymorph Selection. Journal of the American Chemical Society. 2002; 124(50): 14834-14835.http:// dx.doi.org/10.1021/ja0286526

18. Chadwick K, A Myerson, and B Trout. Polymorphic control by heterogeneous nucleation - A new method for selecting crystalline substrates. CrystEngComm. 2011; 13(22).http://dx.doi.org/10.1039 c1ce05871a

19. Kilin DS, OV Prezhdo, and Y Xia. Shape-controlled synthesis of silver nanoparticles: Ab initio study of preferential surface coordination with citric acid. Chemical Physics Letters. 2008; 458(13):113-116.http://dx.doi.org/10.1016/j.cplett.2008.04.046

Copyright:(c) 2012 M. Mohammed, et al. This is an openaccess article distributed under the terms of the Creative Commons Attribution License, which permits unrestricted use, distribution, and reproduction in any medium, provided the original author and source are credited. 